\title{
Growth optimisation of ZnS:Mn thin film phosphors for high intensity miniature electroluminescent displays
}

G. Boutaud, W.M. Cranton, D.C. Koutsogeorgis, R.M. Ranson, C. Tsakonas, C.B. Thomas

Nottingham Trent University

\begin{abstract}
This paper details an investigation into the deposition by RF magnetron sputtering of thin film phosphors specifically for use in laterally emitting thin film electroluminescent (EL) devices. The work presented here is concerned with the use of co-sputtering to optimise luminous efficiency and the use of transient (decay time) measurements to quantify the luminescent quality of the films. Thin films of phosphor were deposited by radio frequency RF magnetron sputtering in a custom built four electrode cluster deposition system. $800 \mathrm{~nm}$ films of $\mathrm{ZnS}$ :Mn were simultaneously cosputtered from $\mathrm{ZnS}$ and $\mathrm{ZnS}: \mathrm{Mn}(1 \mathrm{wt} . \%)$ solid targets. The thin films were deposited at different manganese concentrations by varying the RF power applied to each target. The films were deposited directly onto $100 \mathrm{~mm}$ diameter (100) n-type silicon substrates, or onto a layer of $300 \mathrm{~nm}$ of $\mathrm{Y}_{2} \mathrm{O}_{3}$ to fabricate electroluminescent test devices. Luminescence was characterised via photoluminescent excitation using a 337 $\mathrm{nm}$ pulsed $\mathrm{N}_{2}$ laser, with the $\mathrm{PL}$ optimum obtained at $0.38 \mathrm{ZnS}$ :Mn power ratio. Transient luminescent measurements confirm that the luminescent decay constant decreases with manganese concentration in the $\mathrm{ZnS}: \mathrm{Mn}$ thin films and proves a direct correlation with the Mn dopant concentration. The correlated Mn concentration vs decay constant results are presented as a potential non-destructive (in-situ) technique for characterising this phosphor during deposition.
\end{abstract}

\section{Keywords}

Sputtering; Doping effects; ZnS:Mn; Thin Films; Electroluminescent devices 


\section{Introduction}

Thin film electroluminescence is an emissive display technology that utilises an all solid-state structure for visible light emission suitable for a range of demanding environmental applications. Alternating current thin film electroluminescent (TFEL) devices with $\mathrm{ZnS}: \mathrm{Mn}$ as the active layer based on the Inoguchi model [1] are still the most efficient TFEL devices, and panels based on this technology have been utilised where ruggedness, reliability, high contrast, and high viewing angles are required; such as in niche medical, transport, and military applications [2]. Much work on this technology was undertaken during the 1980s and 1990s, but was overshadowed by the major advances in liquid crystal based display technologies. However, the solid state thin film structure at the heart of TFEL displays provides an inherent ruggedness and potential for low cost manufacture, in comparison to some of the more prevalent, but complex, flat panel displays, and interest in the technology has consequently been rejuvenated recently [3] with the need for higher performance display systems, particularly in avionic applications [4]. One of the negative issues highlighted during the work in the 1990s was the difficulty in controlling the luminescent quality of phosphor films deposited by volume production techniques, such as sputtering. More recently, ACTFEL devices using co-sputtering have been reported [5,6,7,]. The work presented here examines this question and identifies a mechanism to ensure quality monitoring that could be performed in-situ, utilising the inherent luminescent properties of the phosphor thin films.

\section{TFEL Device Technology}

TFEL devices are typically composed of a thin film stack of inorganic layers with the active phosphor thin film sandwiched between two dielectric thin films. The processes of electron emission, acceleration, and phosphor excitation all occur within a 0.5 to 1 
micron-thick solid phosphor film. This design eliminates the need for any vacuum space, making the TFEL displays more durable than some other technologies. The electroluminescent (EL) thin film stack of insulator/ZnS:Mn/insulator is usually grown on glass or on silicon wafers, as in the present work. This paper details an investigation into the deposition by RF magnetron sputtering of thin film phosphors specifically for use in laterally emitting thin film electroluminescent (LETFEL) devices - a novel, high intensity version of TFEL technology that utilises the inherent internal light guiding of the EL thin film stack [8] .

One of the primary factors which governs the efficiency of the $\mathrm{ZnS}: \mathrm{Mn}$ active layer is the Mn dopant concentration. The optimum concentration reported in the literature varies with deposition technique and methods used to characterise, but is typically around $0.5 \mathrm{wt} . \%[9,10,11]$. However some reports have indicated 'optimums' as high as $3 \mathrm{Mn} \mathrm{mol} \%(1.5 \mathrm{wt} . \%)$ [12] or as low as $0.2 \mathrm{wt} . \%$ [13]. Part of the issue related to this inconsistency is the nature of the materials characterisation used - which is often not detailed, and the fact that from our own work we have observed a variation in optimum performance with the age of the sputtering source used, the target supplier, and the annealing temperatures utilised. With volume production in particular, it is the batch to batch deposition variation which is a major concern.

For this work, therefore, we have examined the luminescent behaviour of the films as a function of deposition optimisation, in order to demonstrate the feasibility of cosputtering as a deposition technique - combined with luminescent decay time monitoring that would facilitate the in-situ characterisation of Mn concentration variations and hence could provide a mechanism for positive process control, when using such a co-sputtering method. 


\section{Experimental}

\section{a. Thin film deposition}

Thin films of $\mathrm{ZnS}: \mathrm{Mn}(800 \mathrm{~nm})$ were co-sputtered via the simultaneous sputtering of $\mathrm{ZnS}$ and $\mathrm{ZnS}: \mathrm{Mn}(1 \mathrm{wt} \%$ ) solid targets onto a rotating $100 \mathrm{~mm}$ diameter (100) n-type Si wafer substrate using a custom built Kurt Lesker Torus four-electrode magnetron system represented in Fig. 1. The substrate temperature was maintained at $190^{\circ} \mathrm{C}( \pm$ $10^{\circ} \mathrm{C}$ ) during deposition in $0.40 \mathrm{~Pa}$ of $\mathrm{Ar}$. The vacuum deposition chamber had a base pressure of $1 \times 10^{-4} \mathrm{~Pa}$ and the deposition was followed by an in-situ annealing of $500^{\circ} \mathrm{C}$ for 1 hour in vacuum. Mn concentration in the phosphor films was varied by adjusting the RF power applied to each of the two solid sputtering targets $\mathrm{ZnS}$ (Testbourne Ltd - SuperVac®, Purity 99.99\%). and ZnS:Mn (Testbourne Ltd SuperVac®, ZnS - Mn, 99 - 1 wt.\%, Purity 99.9\%), and a substrate distance of 15 $\mathrm{cm}$ ). The solid $\mathrm{ZnS}: \mathrm{Mn}$ target (1 wt.\% Mn) was produced by Testbourne Ltd (Z19017-M), Purity $99.95 \%$ via their proprietry hot pressing technique. Comparative films were also made from sputtering from a single pressed powder $\mathrm{ZnS}: \mathrm{Mn}$ target (Phosphor Technology - $0.43 \mathrm{Mn}$ wt.\% prepared using a hydraulic target press system). Each target had a $76.2 \mathrm{~mm}$ diameter and a $4.4 \mathrm{~mm}$ thickness. An average of $\pm 3 \%$ thickness variation across the thin films was measured using Dektak $6 \mathrm{M}$ stylus profilometer.

\section{b. Photoluminescence Characterisation}

Time integrated photoluminescence spectra were obtained by exciting the surface of the $\mathrm{ZnS}: \mathrm{Mn}$ thin film phosphor with the $1 \mathrm{~mm}$ diameter beam of a $\mathrm{N}_{2}$ laser $(337 \mathrm{~nm}$ photon energy $=3.68 \mathrm{eV}$, i.e. just above the band gap of $\mathrm{ZnS}$ ). The luminescent PL emission was collected via an optical fibre and the spectra characterised with an Ocean Optics S2000 spectrometer. The maximum intensity of each spectrum was 
recorded for the different $\mathrm{Mn}$ concentrations at the characteristic Mn peak emission wavelength of $585 \mathrm{~nm}$.

\section{c. Luminescent Decay Characterisation}

Using the pulsed laser irradiation from the $\mathrm{N}_{2}$ laser to excite the phosphor, the transient photoluminescence emission from the sample was collected through a UV filter and a monochromator, and measured via an Electrons Tube 9558QB photomultiplier connected to a Gould DSO 4072 digital storage oscilloscope. A value of decay time constant $\tau$ was determined by fitting the transient luminescent data to a

single exponential decay $I=I_{0} e^{\frac{-t}{\tau}}$ where $I_{0}$ is the photoluminescence intensity at the laser irradiation pulse cut off time.

\section{d. Electroluminescence Characterisation}

For comparison of photo and electro-luminescent properties, a series of equivalent EL test devices were fabricated across the range of $\mathrm{Mn}$ concentrations studies. The two sputtered dielectric $\left(\mathrm{Y}_{2} \mathrm{O}_{3}\right)$ thin films $(300 \mathrm{~nm})$ sandwiching the phosphor layer were deposited using the cluster magnetron system of Fig. 1 at a temperature of $190^{\circ} \mathrm{C}$ in 2 mbar of Ar. Following device annealing at $500^{\circ} \mathrm{C}$ for 1 hour in vacuum, a sputtered indium tin oxide transparent conducting layer was deposited at $200 \mathrm{~nm}$ thick as the top transparent electrode layer (in a separate deposition system). Luminous intensities were measured using a LS1 10 luminance meter coupled to a computer controlled system by applying a sinusoidal waveform to excite the thin film electroluminescent devices. 


\section{Results}

It is important to mention that all the films examined were similar in terms of thickness, adhesion and visual appearance. In addition, for these films the variation was in doping, and no change in XRD spectra was observed between samples.

The peak photoluminescent intensity (at $585 \mathrm{~nm}$ ) as a function of the $\mathrm{Mn}$

concentration is shown in Fig. 2. The target power was kept fixed at $50 \mathrm{~W}$ for the $\mathrm{ZnS}$ electrode and varied from 15 to $50 \mathrm{~W}$ for the $\mathrm{ZnS}: \mathrm{Mn}$ electrode, while the single pressed target was sputtered at a power of $80 \mathrm{~W}$.

The photoluminescent optimum was obtained at a 0.38 ratio of the if power supplied

to the two targets (power ratio defined as: $P=\frac{P_{\text {doped }}}{P_{\text {doped }}+P_{\text {undpoed }}}, P_{\text {undoped }}$ was kept constant while $P_{\text {doped }}$ was varied), which is closely related to the nominal final Mn concentration in the film (i.e. $0.38 \mathrm{wt} \% \mathrm{Mn}$ ). The actual Mn concentration will be verified with RBS and SIMS analysis, but initial trials using electron probe wavelength dispersive $\mathrm{x}$-ray analysis have indicated that the expected concentrations are $\pm 10 \%$ of the value predicated by the ratio of the rf-power supplied to the two targets. Indeed, for this study, it is the nominal Mn concentration that is important, since the use of luminescence decay can then be implemented for 'optimum' dopant monitoring in the actual films, rather than relying on subsequent indirect and destructive materials characterisations.

By applying $\mathrm{N}_{2}$ laser excitation pulses of $4 \mathrm{~ns}$ width and a repetition rate of $20 \mathrm{~Hz}$, the photoluminescent emission was recorded and noise reduction was performed by digitally averaging the response over 256 cycles. The photoluminescent decay curves from 6 different concentrations are illustrated in Fig. 3. The photoluminescence rises sharply with the application of the excitation pulse and then decays exponentially. 
For this work, the double exponential decay of $\mathrm{ZnS}$ :Mn can be approximated to be composed of a first quick single exponential decay (directly after the laser pulse) within the first 0.05 to $0.15 \mathrm{~ms}$, followed by a slower single exponential decay within the period $0.15 \mathrm{~ms}$ to $1 \mathrm{~ms}$. For the purpose of this $\mathrm{Mn}$ concentration comparison, the decay constant was calculated using a single exponential fit to the luminescent decay during a fixed time domain of 0.2 to $0.5 \mathrm{~ms}$ after the end of the laser pulse. This period was chosen because of its good curve fitting (with a $0.93<\mathrm{R}^{2}<0.99$ ), its measurement consistency and the elimination of interference from the laser pulse.

The results showing the average decay constant as a function of Mn concentration are represented in Fig. 4. These results clearly show the influence of the manganese concentration on the decay constant. With increasing the concentration of $\mathrm{Mn}^{2+}$ ions in the host $\mathrm{ZnS}$ lattice, the after-glow period (when the excitation has ceased) decreases. This confirms previous results $[9,14,15]$, and is indicative of the effect that is associated with $\mathrm{Mn}-\mathrm{Mn}$ ion interactions leading to non-radiative decay probability increase. What is interesting and potentially of value in process control systems, is the clear demonstration presented in the results of Fig. 4 that there is a direct correlation between the PL luminescent decay constant measured in this way, and the nominal Mn concentration - hence optimum deposition conditions

For electroluminescent evaluation of co-sputtered films, a $1 \mathrm{kHz}$ sinusoidal waveform voltage was applied to the test TFEL devices and luminance was recorded as a function of drive voltage applied, as shown in Fig. 5 with six different $\mathrm{Mn}$ concentrations. Fig. 6 shows the operating luminance (defined in this work as the threshold voltage, $\mathrm{V}_{\text {th }}$, plus $50 \mathrm{~V}$ ) vs $\mathrm{Mn}$ concentration. The results show that with these films, the 'optimum' electroluminescence is obtained for a similar nominal $\mathrm{Mn}$ 
concentration $(\sim 0.36 \mathrm{Mn} w \mathrm{w} . \%)$ compared to the photoluminescence results achieved from a different set of wafers, which also compares favourable to results obtained previously which showed an optimum at $0.43 \mathrm{wt} . \%$ [15].

\section{Discussion and Conclusions}

Ac excited electroluminescence within $\mathrm{ZnS}: \mathrm{Mn}$ thin films is known to occur as a result of the substitutional incorporation of $\mathrm{Mn}^{2+}$ into the host lattice, where a $\mathrm{Zn}$ vacancy is occupied by the dopant ion. The $2.1 \mathrm{eV} 3 \mathrm{~d}$ shell transition that is responsible for the characteristic $585 \mathrm{~nm} \mathrm{Mn} \mathrm{emission} \mathrm{is} \mathrm{spin} \mathrm{and} \mathrm{parity} \mathrm{forbidden} \mathrm{for}$ the isolated Mn atom, and can only occur when the field is modified within the lattice. A correctly sited $\mathrm{Mn}$ ion is therefore termed an emitting centre and may be excited by impact excitation, which is thought to dominate in EL, or by energy exchange from electron-hole recombination as produced by PL excitation. However, during the early work on TFEL in the 1980 s, it was reported that less than $1 \%$ of the dopant atoms are participating in the emission process, but that the cross-section for excitation is high, being of the order of the geometric cross-section $-1 \times 10^{16} \mathrm{~cm}^{2}[16]$. This implies that the majority of dopant atoms are not correctly sited within the lattice. The nature of the dopant environment is thus highly influential on the luminescent properties, and is strongly affected by deposition conditions - particularly annealing - as well as by the actual doping density.

The rapid rise in luminescent emission by both PL and EL as Mn concentrations is increased towards the optimum (here shown as $0.3-0.4 \mathrm{wt} . \%$ ) is related to the concomitant increase in available emitting centres - i.e. substitutional Mn ions. Beyond the optimum, the rapid fall off in luminescent emission is demonstrated to be correlated to the corresponding decrease in luminescent decay time, which can be considered to be due to an increase in non-radiative decay probability associated with 
Mn-Mn interactions $[14,17,18,19]$. The decay results shown in Fig. 4 give clear indication that a suitable measurement of the transient luminescence is a good indicator of the internal effective doping concentration and thus provides a potential quality monitoring technique that could be implemented as an in-situ growth monitor The results show this to be true for the range of doping density up to $0.8 \mathrm{wt} . \%$.

While the link between decay time and Mn doping has been indicated in the past, this is the first demonstration of a clear correlation of the nominal optimised $\mathrm{Mn}$ concentration to a measurable parameter that would be an effective performance monitor. Changes in both dopant concentration and environment would be reflected in the position on the curve of Fig. 4 and hence could be utilised to change deposition and/or annealing conditions in order to compensate for the known variation in sputtering that results with target age and batch changes.

Furthermore, a variation in nominal Mn concentration between depositions results in a corresponding variation in the electroluminescent intensity (as shown in Fig. 6) demonstrates the correlation between deposition control on the nominal $\mathrm{Mn}$ concentration and resultant performance.

In summary, the use of a co-sputtering technique for the deposition of luminescent phosphor thin films of $\mathrm{ZnS}: \mathrm{Mn}$ has been demonstrated with results showing that an optical measurement technique could be utilised to monitor the effective $\mathrm{Mn}$ concentration and thus provide a process control tool to ensure optimised batch to batch film deposition. These films are now being investigated for use in high intensity TFEL devices for sunlight readable indicator display applications. 


\section{Acknowledgements}

This work forms part of a collaborative research programme - Sunlight Readable Electroluminescent Displays which is supported by the UK Technology Strategy Board and by a Nottingham Trent University Vice Chancellor's research student bursary.

\section{References}

[1] T. Inoguchi, M. Takeda, Y. Yakihara, Y. Nakata, and Y. M., Proceedings of the S.I.D 74 (1974) 84-85.

[2] H. Inuzuka, T. Yamauchi, Y. Hattori, M. Katayama, N. Itou, IDMC 2000 (2000) paper 12.1 .

[3] A.R. Kmetz, Information Display 19, part 3, (2003) 26-33.

[4] W.M. Cranton, R. Stevens, D.C. Koutsogeorgis, R.M. Ranson, A. Needham, M. Thorne, A. Magon; 14th International Conference on Vehicles and Photons, Digest of technical papers, Detroit (2007) 27.

[5] H. Fukada, S. Matsui, T. Miyata, and T. Minami, Journal of Vacuum Science \& Technology A (Vacuum, Surfaces, and Films) 26, n 4, (2008) 944-8.

[6] W. Glass, A. Kale, N. Shepherd, M. Davidson, D. DeVito, and P. H. Holloway, Journal of Vacuum Science \&amp; Technology A (Vacuum, Surfaces, and Films) 25 (2007) 492.

[7] Z. Ding, Z. Yu, and W. Xue, Proc. SPIE, Beijing 6624 (2008) 66241.

[8] W.M. Cranton, R. Stevens, C.B. Thomas, Information Display (18) (2002) 2225.

[9] N.A. Vlasenko, S.A. Zynio, and Y.V. Kopytko, Physica Status Solidi A 29, (1975) 671.

[10] A. J. Warren, C. B. Thomas, and P. R. C. Stevens, Journal of Physics D (Applied Physics) 16 (1983) 225.

[11] K. Takamichi, A. Nitta, K. Tanaka, and M. Aozasa, Memoirs of the Faculty of Engineering, Osaka City University 43, (2002) 1.

[12] S. Schon, M. Chaichimansour, W. Park, E.W. Thomas, T. Yang, B.K. Wagner, and C. J. Summers, Organic Light-Emitting Materials and Devices II (Proceedings of Spie - the International Society for Optical Engineering, V. 3476), San Diego (1998) 67.

[13] V. Marrello and A. Onton, IEEE Transactions on Electron Devices ED-27 (1980) 1767.

[14] H. S. Bhatti, R. Sharma, and N. K. Verma, Physica B: Condensed Matter 382 (2006) 38 . 
[15] W. M. Cranton, PhD. Thesis, University of Bradford (1995).

[16] D.H. Smith, J. Lum. 23 (1981) 209.

[17] B. Selle, Physica Status Solidi 5 (1964) 649.

[18] R. Tornqvist, J. Appl. Phys. 54 (1983) 7.

[19] A. Fuh, R. P. Gallinger, P. Schuster, J. Adolph, and O. Caporaletti, Thin Solid Films 207 (1992) 202. 


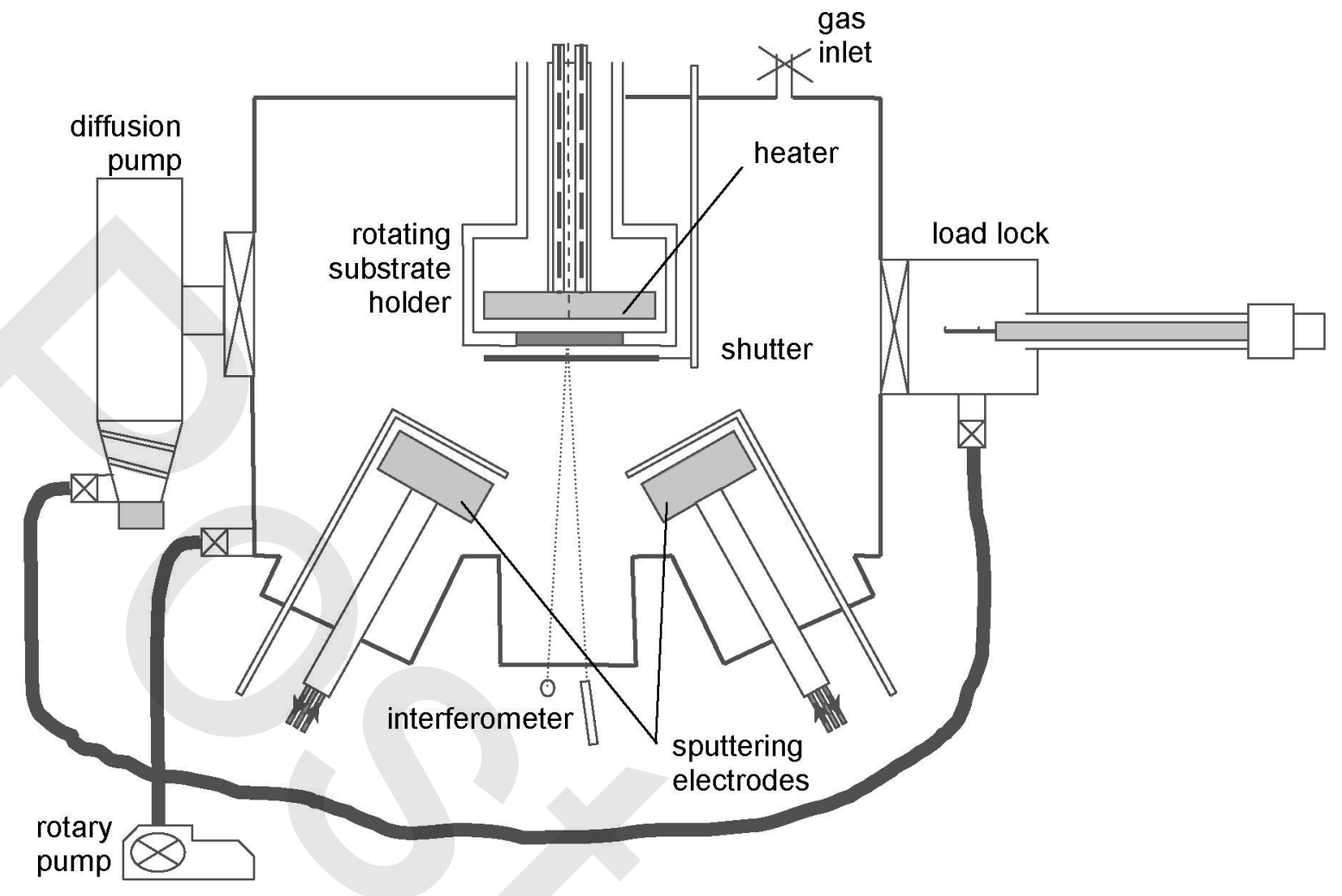

Fig. 1. Schematic cross section of RF magnetron cluster deposition system. 


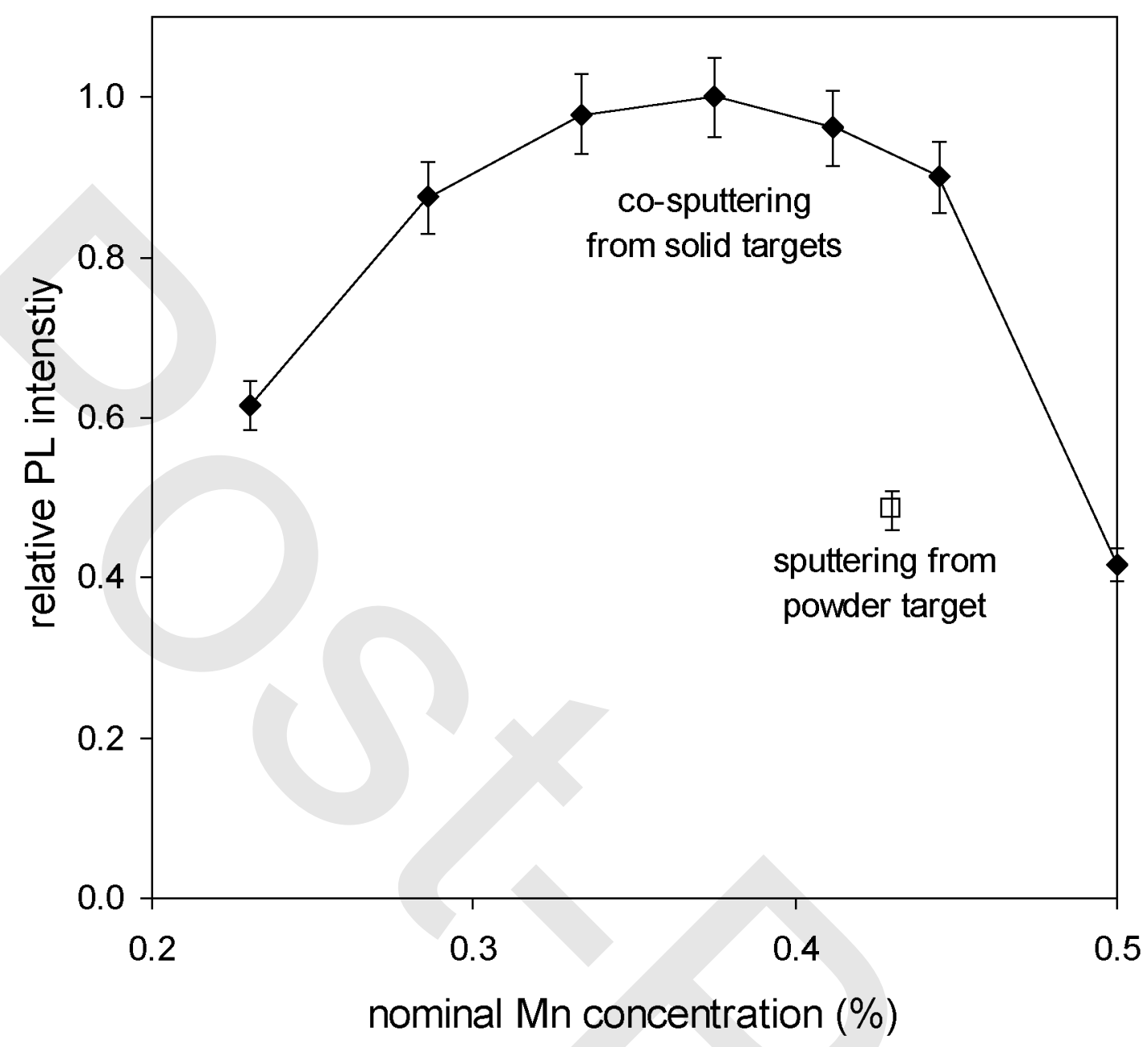

Fig. 2. Integrated time photoluminescence intensity as a function of nominal Mn concentration (represented by co-sputtering power ratio applied) for $800 \mathrm{~nm}$ thick films of $\mathrm{ZnS}: \mathrm{Mn}$ on $\mathrm{Si}$ at room temperature, with excitation from an $\mathrm{N}_{2}$ laser at $337 \mathrm{~nm}$. 


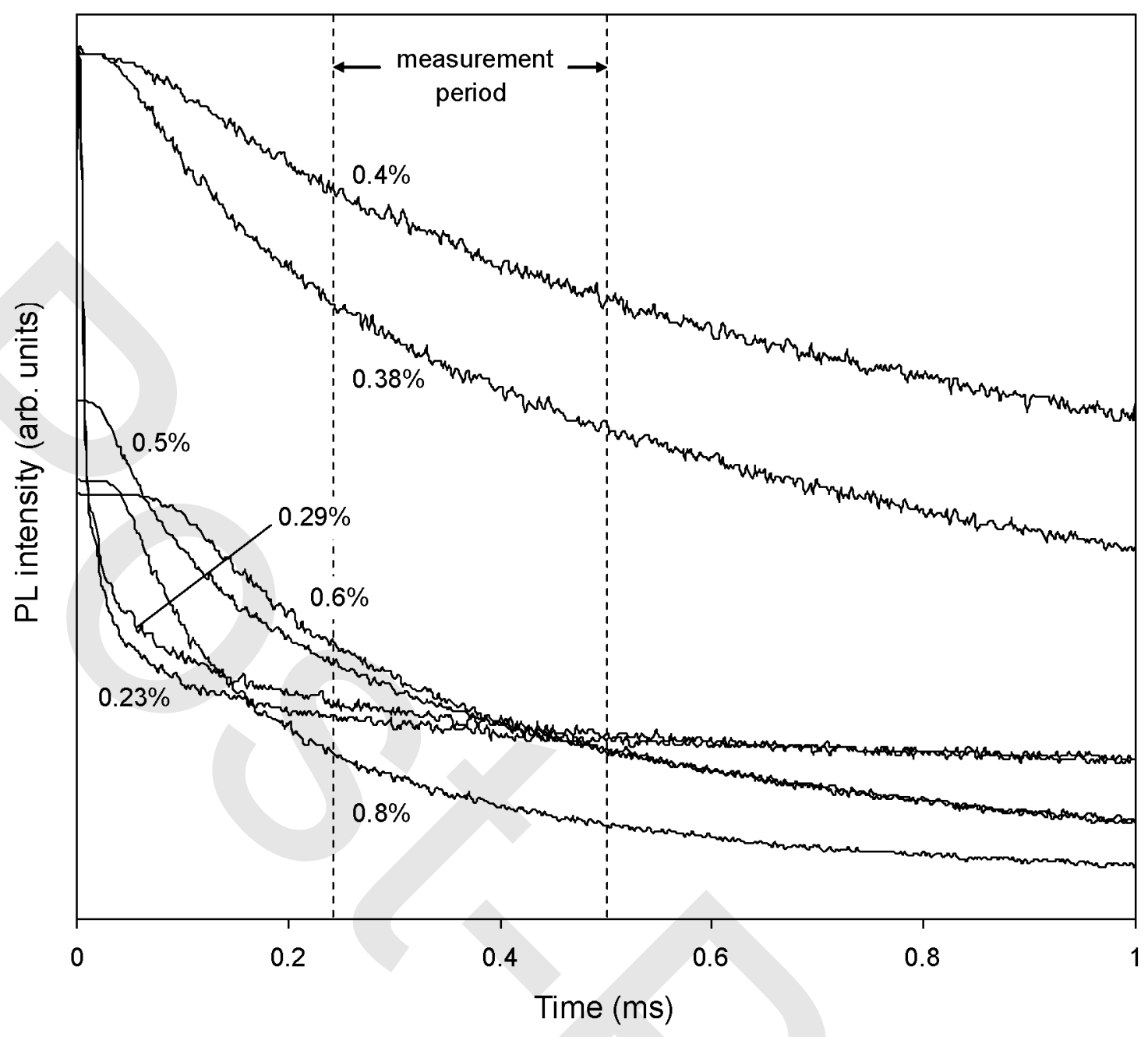

Fig. 3. Photoluminescence decay curves of $800 \mathrm{~nm}$ thick films of $\mathrm{ZnS}: \mathrm{Mn}$ on Si at room temperature at different nominal Mn concentrations (represented by cosputtering power ratio applied). Dashed lines indicate the time domain used for decay constant determination. 


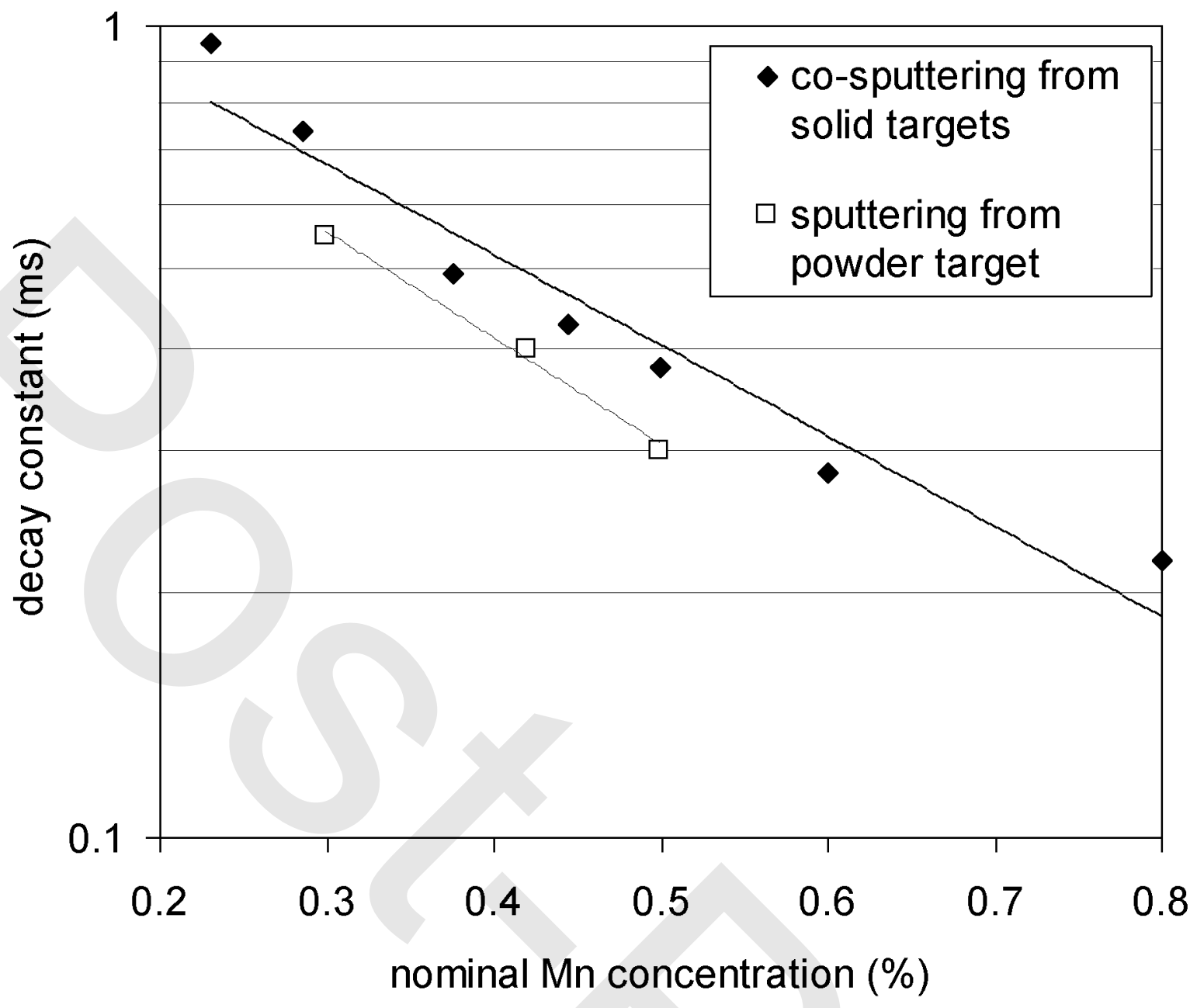

Fig. 4. Photoluminescence decay constant as a function of nominal Mn concentration (represented by co-sputtering power ratio applied) for $800 \mathrm{~nm}$ thick films of $\mathrm{ZnS}: \mathrm{Mn}$ on $\mathrm{Si}$ at room temperature. Also shown are results from a previous study for comparison with co-sputtered powder source targets of $\mathrm{ZnS}$ and $\mathrm{ZnS}: \mathrm{Mn}(1 \mathrm{wt} \%)$ from ref [18]. 


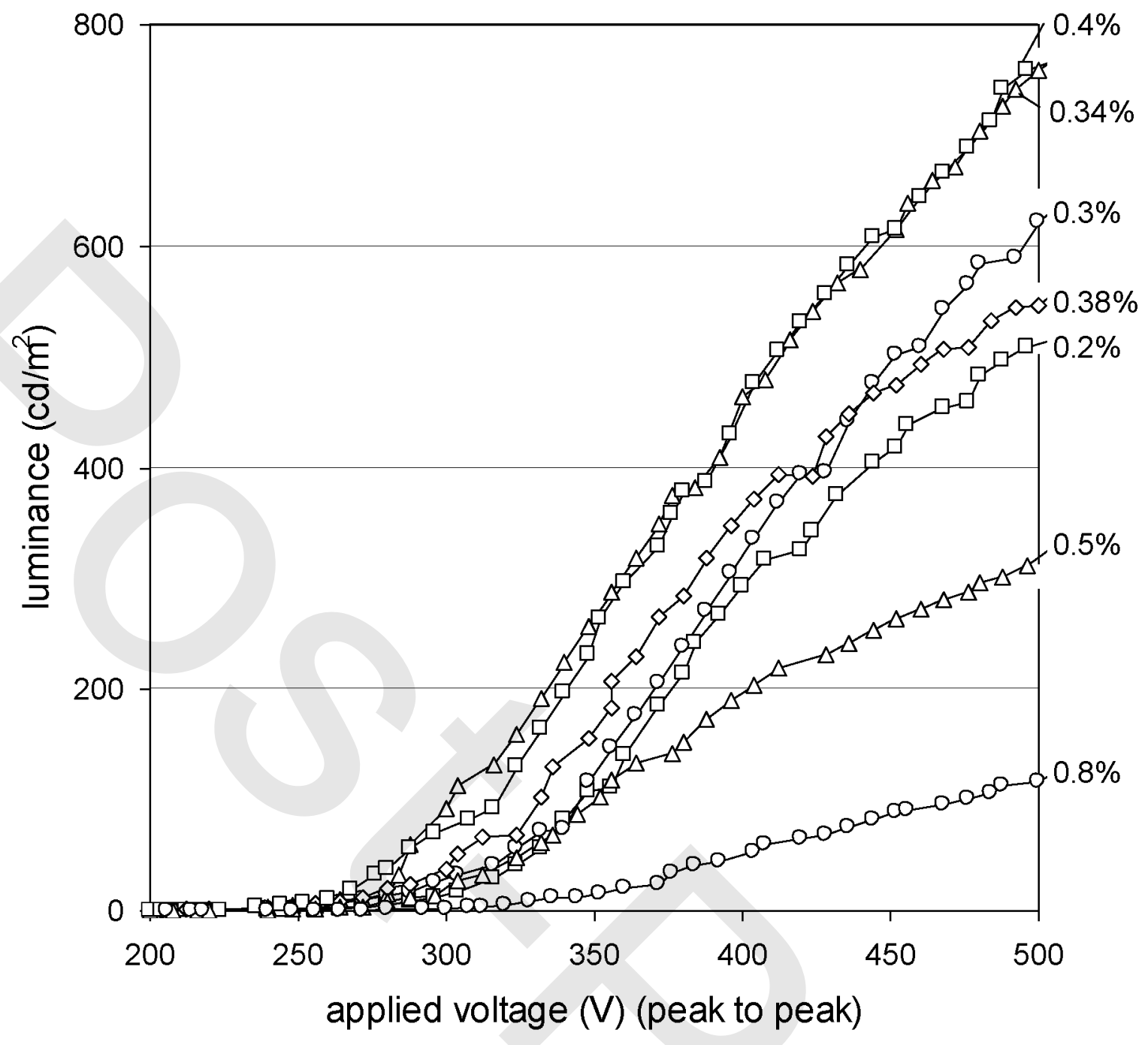

Fig. 5. Luminance vs drive voltage characteristics of $\mathrm{ZnS}: \mathrm{Mn}$ based TFEL test devices as a function of the co-sputtering power ratio used to vary the nominal $\mathrm{Mn}$ concentration. 


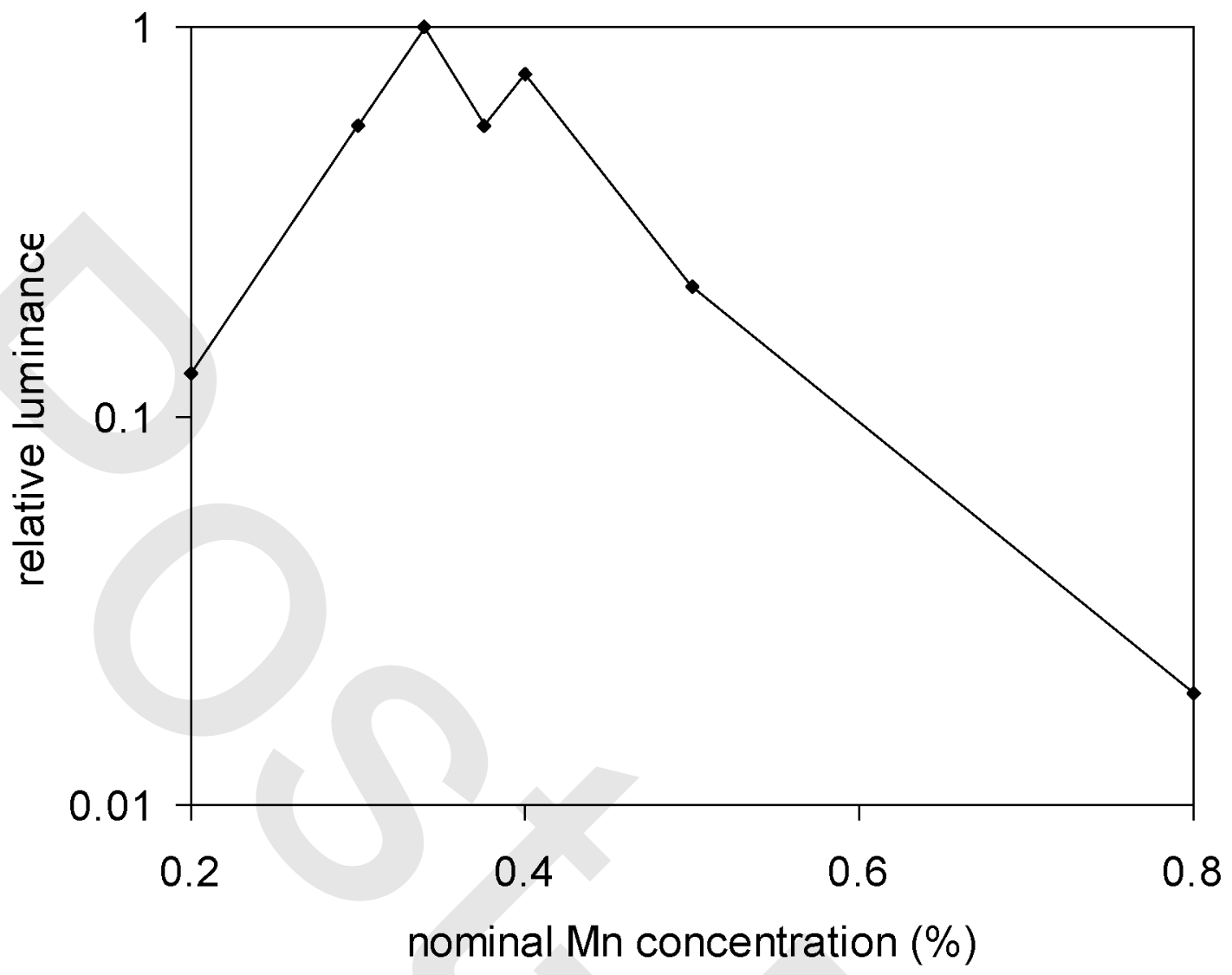

Fig. 6. Electroluminescence luminance value vs nominal Mn concentration (represented by co-sputtering power ratio applied) at operating voltage $\left(\mathrm{V}_{\text {th }}+50 \mathrm{~V}\right.$, ground to peak) for TFEL devices. 